\section{Leveraging the Use of mHealth in Patients with Depression among Low- and Middle- Income Countries}

Sir,

Low- and middle-income countries (LMIC) usually suffer from burden of mental disorders, fundamentally because of underdeveloped healthcare systems. ${ }^{1}$ Around $90 \%$ of people residing in LMICs having mental disorders receive no mental healthcare services. ${ }^{1}$ Over the past few decades, depression is found to be a highly prevalent mental health disorder. ${ }^{2}$ In developing countries, 50.8 million people have depression, while 10 to $44 \%$ suffer from depressive disorders. ${ }^{2,3}$ The situation is quite serious in Karachi, Pakistan, where approximately $36 \%$ of people suffer from depression, which is in alignment to the World Health Organization (WHO) estimates. ${ }^{2}$ It is estimated to be the $10^{\text {th }}$ leading cause of disability-adjusted life years (DALYs) lost both nationally and globally. ${ }^{4}$

LMICs are faced with significant barriers that limit access to healthcare services, including high illiteracy levels, lack of finances, lack of awareness (stigma and social exclusion); and dearth of mental healthcare professionals that further add to the disease burden. ${ }^{1}$ The proportion of mental health professionals to the general population is expected to be nearly 200 times higher in high-income nations than resource-limited countries. ${ }^{1}$ In countries like Chad, Liberia, and Rwanda, only one or two psychiatrists are available for the whole country! $!^{1,5}$ Moreover, less than US $\$ 2$ per capita per annum is spent on mental health services in LMICs. ${ }^{5}$ In Pakistan, healthcare expenditure budget is less than $1 \%$ of the gross national product (GNP); and unfortunately, only $0.4 \%$ of the total budget is allocated to mental health, which significantly affects the underprivileged communities of the society. This, in turn reflects, that the patients have to pay out of their pockets for their mental health treatment as the coverage by companies is limited with only a few insurance companies in the market with low premium policies. ${ }^{2}$ In order to cater this high demand of mental health, innovative approaches that are accessible, less time-consuming, and cost-effective are urgently needed.

Leveraging the digital technology, i.e. mobile phones, may indicate a viable solution for dealing with high burden of mental disorders. Mobile health (mHealth) refers to the utilisation of mobile technology in healthcare delivery system. ${ }^{1,6}$ Mobile phones have breached all the geospatial boundaries, particularly in LMICs where accessibility is a major concern. Recently, WHO has designed a "step-by-step" application that offers psychoeducation to depressive patients and provides training in behavioural activation. ${ }^{5}$ Similarly, another application entitled "global action intervention guide" launched by WHO, recently scaled up in 90 countries, is used to build capacity of physicians in LMICs to diagnose and manage high-risk mental health conditions like depression based on standard guidelines and protocols. ${ }^{6}$ Thus, mHealth technology can provide accurate and timely diagnosis, and simultaneously improving prognosis of depression.

LMICs continue to grapple with scarce resources and wide treatment gap; mHealth can facilitate in bridging this gap and offer ways to overcome barriers, irrespective of geopolitical borders, time zones, and cost. It facilities in building capacity of mental health workforce as well as strengthens the existing healthcare system. Furthermore, a public-private partnership approach is needed to integrate mHealth in mental healthcare services and overall in healthcare systems, particularly in LMICs.

\section{CONFLICT OF INTEREST:}

Authors declared no conflict of interest.

\section{AUTHORS' CONTRIBUTION:}

NA: Contributed in drafting the entire manuscript. AK: Finalized the draft and has given critical feedback.

\section{REFERENCES}

1. Naslund JA, Aschbrenner KA, Araya R, Marsch LA, Unützer J, Patel V, et al. Lancet Psychiatry 2017; 4:486-500.

2. Ahmed B, Enam SF, Iqbal Z, Murtaza G, Bashir S. Depression and anxiety a snapshot of the situation in Pakistan. Int J Neurosci and Behav Sci 2016; 4:32-6.

3. World Health Organization (WHO, 2001). World Health Report Geneva, Switzerland.

4. World Health Organization (WHO, 2019). Depression available online:http://www.emro.who.in/health-topics/depression/ index.html.

5. Carswell K, Harper-Shehadeh M, Watts S, van't Hof E, Ramia JA, Heim E, et al. Step-by-step a new WHO digital mental health intervention for depression. Mhealth 2018; 4:34.

6. Keynejad RC, Dua T, Barbui C, Thornicroft G. WHO mental health gap action programme (mhGAP) intervention guide a systematic review of evidence from low and middle-income countries. Evid Based Ment Health 2018; 21:30-4.

Naureen Akber Ali ${ }^{1}$ and Adeel Khoja ${ }^{2}$

${ }^{1}$ School of Nursing and Midwifery, The Aga Khan University Hospital, Karachi, Pakistan

${ }^{2}$ Department of Medicine, The Aga Khan University, Karachi, Pakistan

Correspondence to: Dr. Naureen Akber Ali, School of Nursing and Midwifery, The Aga Khan University Hospital, Stadium

Road, Karachi, Pakistan

E-mail: naureen.akberali@aku.edu

Received: June 21, 2019; Revised: July 10, 2019;

Accepted: August 08, 2019

DOI: https://doi.org/10.29271/jcpsp.2020.04.458 